\title{
ERRATA
}

Artigo originalmente publicado com nome da instituição de Luciano Brito Rodrigues errado

\section{Falhas na utilização de poliacetal e poliamida em forma de haste intramedular bloqueada para imobilização de fratura femural induzida em bovinos jovens}

Failures in the use of polyacetal and polyamide in the form of intramedullary interlocking nail for immobilization of induced femoral fracture in young cattle

\author{
Odael Spadeto Junior ${ }^{\mathrm{I}, \mathrm{II} *}$ Rafael Resende Faleiros ${ }^{\mathrm{I}}$ Geraldo Eleno Silveira Alves $^{\mathrm{I}}$ \\ Estevam Barbosa de Las Casas ${ }^{\mathrm{III}}$ Luciano Brito Rodrigues ${ }^{\mathrm{III}}$, IV Bruno Zambelli Loiacono \\ Fabiane Cassou
}

Para html ou PDF, acesse, respectivamente:

http://www.scielo.br/scielo.php?script=sci_arttext\&pid=S0103-84782010000400025\&lng=en\&nrm=iso\&tlng=pt

ou

http://www.scielo.br/pdf/cr/v40n4/a521cr2301.pdf

We sugest that subscribers photocpy this correction and insert the copy at the appropriate place where the article originally appeared.

Sugerimos que uma fotocópia das correções seja inserida na página correta onde o artigo apareceu originalmente.

IPrograma de Pós-graduação em Ciência Animal, Departamento de Clínica e Cirurgia Veterinária, Universidade Federal de Minas Gerais (UFMG). Av. Antonio Carlos, 6627, 30123-970, Belo Horizonte, MG, Brasil. E-mail: odaeljr@ hotmail.com. Autor para correspondência.

"Hospital Veterinário, Centro Universitário de Vila Velha (UVV), Vila Velha, ES, Brasil.

'IIPrograma de Pós-graduação em Engenharia Mecânica, Escola de Engenharia, UFMG, Belo Horizonte, MG, Brasil.

${ }^{\text {IV }}$ Departamento de Tecnologia Rural e Animal, Universidade Estadual do Sudoeste da Bahia, Itapetinga, BA, Brasil. 